\title{
A Stabilized Lagrange Multiplier Method for the Finite Element Approximation of Frictional Contact Problems in Elastostatics
}

\author{
V. Lleras ${ }^{1}$ \\ Laboratoire de mathématiques, Université de Besançon, CNRS UMR 6623, 16 route de Gray \\ 25030 Besançon Cedex, France
}

\begin{abstract}
In this work we consider a stabilized Lagrange multiplier method in order to approximate the Coulomb frictional contact model in linear elastostatics. The particularity of the method is that no discrete inf-sup condition is needed. We study the existence and the uniqueness of solution of the discrete problem.
\end{abstract}

Key words: unilateral contact, Coulomb's friction law, finite elements, mixed method, stabilization.

AMS subject classification: $65 \mathrm{~N} 30,74 \mathrm{M} 15$.

\section{Introduction and notation}

The numerical approximation of frictional contact problems occurring in structural mechanics is generally achieved using the finite element method (see [23, 26, 36, 38, 49]). An important aspect in these simulations consists of choosing finite element methods which are both easy to implement in practice and accurate from a theoretical point of view. The frictionless unilateral contact problem (or the equivalent scalar valued Signorini problem) shows the nonlinearity on the boundary corresponding to the non-penetration of the materials on the contact area which leads to a variational inequality of the first kind. When considering friction in addition to the contact model, there are supplementary nonlinearities which have to be taken into account. We consider in what follows

\footnotetext{
${ }^{1}$ E-mail: vanessa.lleras@univ-fcomte.fr
} 
the simplest model: linear elasticity, small strains and Coulomb friction. Although there exist simplified and/or different models: Tresca's friction, normal compliance...(see [36, 47]), we consider the so-called static friction problem introduced in $[19,20]$ which roughly speaking corresponds to an incremental problem in the time discretized quasi-static model. Let us mention that a detailed study of several mixed finite element methods for frictionless and frictional contact problem can be found in $[25,26]$. We can find also numerical analysis and convergence analysis in [16, 33].

For this elementary model without friction, some results were improved and/or generalized in many directions using different kind of multipliers [9, 10, 18], quadratic finite elements [8, 32] or an augmented Lagrangian [14]. In fact, any of the mixed methods cited above need an inf-sup condition (see $[3,12,13])$ and a number of convenient choices are ruled out by this condition. In the present work we consider a mixed finite element method which does not require an infsup condition. Such methods which provide stability of the multiplier by adding supplementary terms in the weak formulation have been originally introduced and analyzed in $[4,5]$. The great advantage of such methods compared to original one in [3] is that the finite element spaces on the primal and dual variables can be chosen independently. In penalty methods, the penetration between two contacting boundaries is introduced and the normal contact force is related to the penetration by a penalty parameter $([2,45])$. Moreover, contrary to penalization techniques, in the method of Lagrangian multipliers, the stability is improved without compromising the consistency of the method. Later, the connection was made in [48] between the stabilized method of Barbosa and Hughes [4, 5] and the former one of Nitsche [44]. The studies in [4, 5] were generalized to a variational inequality framework in [6] (Signorini type problems among others). This method has also been extended to interface problems on nonmatching meshes in [7, 24] and more recently for bilateral (linear) contact problems in [29]. My aim in this paper is to extend the work of Hild and Renard in [34] to the more general and currently used Coulomb's frictional contact model but without convergence analysis.

The paper is outlined as follows. In section 2, we introduce the equations modelling the frictional unilateral contact of a linear elastic body with a rigid foundation under the small strains hypothesis. We write the problem using a mixed formulation where the unknowns are the displacement field in the body and the frictional contact pressures on the contact area. In section 3, we propose an extension of "Barbosa-Hughes-Nitsche's" concept to the frictional contact problem and we study the properties of the discrete problem.

Finally, we introduce some useful notations and several functional spaces. In what follows, bold letters like $\mathbf{u}, \mathbf{v}$, indicate vector or tensor valued quantities, while the capital ones (e.g., $\mathbf{V}, \mathbf{K}, \ldots$ ) represent functional sets involving vector fields. As usual, we denote by $\left(L^{2}(.)\right)^{d}$ and by $\left(H^{s}(.)\right)^{d}, s \in \mathbb{R}, d=1,2,3$ the Lebesgue and Sobolev spaces in one, two or three space dimensions (see [1]). The usual norm of $\left(H^{s}(D)\right)^{d}$ (dual norm if $s<0$ ) is denoted by $\|\cdot\|_{s, D}$ and we keep the same notation when $d=1, d=2$ or $d=3$. Finally the notation $a \lesssim b$ means here and below that there exists a positive constant $C$ independent of $a$ and $b$ (and of the meshsize of the triangulation) such that $a \leq C b$. The notation $a \sim b$ means that $a \lesssim b$ and $b \lesssim a$ hold simultaneously. 


\section{The frictional contact problem in elasticity}

We consider the deformation of an elastic body occupying, in the initial unconstrained configuration, a domain $\Omega$ in $\mathbb{R}^{2}$ where plane small strain assumptions are assumed. The Lipschitz boundary $\partial \Omega$ of $\Omega$ is polygonal and we suppose that $\partial \Omega$ consists in three nonoverlapping parts $\Gamma_{D}, \Gamma_{N}$ and the frictional contact boundary $\Gamma_{C}$ with meas $\left(\Gamma_{D}\right)>0$ and meas $\left(\Gamma_{C}\right)>0$. The normal unit outward vector on $\partial \Omega$ is denoted $\mathbf{n}=\left(n_{1}, n_{2}\right)$ and we choose as unit tangential vector $\mathbf{t}=\left(-n_{2}, n_{1}\right)$. The body is clamped on $\Gamma_{D}$ for the sake of simplicity. It is subjected to volume forces $\mathbf{f}=\left(f_{1}, f_{2}\right) \in\left(L^{2}(\Omega)\right)^{2}$ and to surface loads $\mathbf{g}=\left(g_{1}, g_{2}\right) \in\left(L^{2}\left(\Gamma_{N}\right)\right)^{2}$. The contact boundary is supposed to be a straight line segment. In its initial stage, the body is in contact on $\Gamma_{C}$ with a rigid foundation (the extension to two elastic bodies in contact can be easily made, at least for small strain models). The contact is assumed to be frictional and the stick, slip and separation zones are not known in advance and we suppose that the unknown final contact zone after deformation will be included into $\Gamma_{C}$. We denote by $\mu \geq 0$ the given friction coefficient on $\Gamma_{C}$.

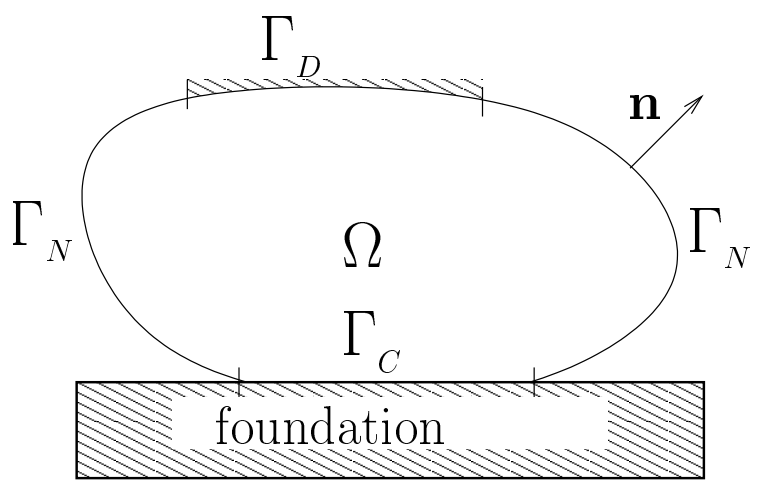

Figure 1: Setting of the problem. The domain $\Omega$ and its boundary divided in three open disjoint parts: $\Gamma_{D}, \Gamma_{N}$ and $\Gamma_{C}$.

The unilateral contact problem with Coulomb's friction law in linear elasticity consists in finding the displacement field $\mathbf{u}: \Omega \rightarrow \mathbb{R}^{2}$ verifying the equations and conditions (2.1)-(2.6):

$$
\begin{array}{rll}
\operatorname{div} \boldsymbol{\sigma}(\mathbf{u})+\mathbf{f} & =\mathbf{0} \quad \text { in } \Omega, \\
\boldsymbol{\sigma}(\mathbf{u}) & =\mathbf{A} \varepsilon(\mathbf{u}) \quad \text { in } \Omega, \\
\mathbf{u} & =\mathbf{0} & \text { on } \Gamma_{D}, \\
\boldsymbol{\sigma}(\mathbf{u}) \mathbf{n} & =\mathbf{g} & \text { on } \Gamma_{N},
\end{array}
$$

where $\boldsymbol{\sigma}=\left(\sigma_{i j}\right), 1 \leq i, j \leq 2$, stands for the stress tensor field and $\operatorname{div}$ denotes the divergence operator of tensor valued functions. The notation $\varepsilon(\mathbf{v})=\left(\boldsymbol{\nabla} \mathbf{v}+\boldsymbol{\nabla} \mathbf{v}^{T}\right) / 2$ represents the linearized strain tensor field and $\mathbf{A}$ is the fourth order symmetric elasticity tensor having the usual uniform ellipticity and boundedness property. For any displacement field $\mathbf{v}$ and for any density of surface 
forces $\boldsymbol{\sigma}(\mathbf{v}) \mathbf{n}$ defined on $\partial \Omega$, we adopt the following notations:

$$
\mathbf{v}=v_{n} \mathbf{n}+v_{t} \mathbf{t} \quad \text { and } \quad \boldsymbol{\sigma}(\mathbf{v}) \mathbf{n}=\sigma_{n}(\mathbf{v}) \mathbf{n}+\sigma_{t}(\mathbf{v}) \mathbf{t} .
$$

On $\Gamma_{C}$, the three conditions representing unilateral contact are given by:

$$
u_{n} \leq 0, \quad \sigma_{n}(\mathbf{u}) \leq 0, \quad \sigma_{n}(\mathbf{u}) u_{n}=0
$$

and the Coulomb friction law is summarized by the following conditions:

$$
\left\{\begin{array}{l}
u_{t}=0 \Longrightarrow\left|\sigma_{t}(\mathbf{u})\right| \leq \mu\left|\sigma_{n}(\mathbf{u})\right| \\
u_{t} \neq 0 \Longrightarrow \sigma_{t}(\mathbf{u})=-\mu\left|\sigma_{n}(\mathbf{u})\right| \frac{u_{t}}{\left|u_{t}\right|}
\end{array}\right.
$$

The weak variational formulation of (2.1)-(2.6) uses the Hilbert spaces

$$
\mathbf{V}=\left\{\mathbf{v} \in\left(H^{1}(\Omega)\right)^{2}: \quad \mathbf{v}=\mathbf{0} \text { on } \Gamma_{D}\right\}, \quad W_{n}=\left\{\left.v_{n}\right|_{\Gamma_{C}}: \mathbf{v} \in \mathbf{V}\right\}, W_{t}=\left\{\left.v_{t}\right|_{\Gamma_{C}}: \mathbf{v} \in \mathbf{V}\right\}
$$

and their topological dual spaces $\mathrm{V}^{\prime}, W_{n}^{\prime}, W_{t}^{\prime}$, endowed with their usual norms. Since $\Gamma_{C}$ is a straight line segment, we have $H_{00}^{1 / 2}\left(\Gamma_{C}\right) \subset W_{n} \subset H^{1 / 2}\left(\Gamma_{C}\right)\left(\right.$ resp. $H_{00}^{1 / 2}\left(\Gamma_{C}\right) \subset W_{t} \subset H^{1 / 2}\left(\Gamma_{C}\right)$ ) which implies $W_{n}^{\prime} \subset H^{-1 / 2}\left(\Gamma_{C}\right)$ (resp. $W_{t}^{\prime} \subset H^{-1 / 2}\left(\Gamma_{C}\right)$ ). Classically, $H^{1 / 2}\left(\Gamma_{C}\right)$ is the space of the restrictions on $\Gamma_{C}$ of traces on $\partial \Omega$ of functions in $H^{1}(\Omega)$, and $H^{-1 / 2}\left(\Gamma_{C}\right)$ is the dual space of $H_{00}^{1 / 2}\left(\Gamma_{C}\right)$ which is the space of the restrictions on $\Gamma_{C}$ of functions in $H^{1 / 2}(\partial \Omega)$ vanishing outside $\Gamma_{C}$. We refer to [39] and [1] for a detailed presentation of trace operators and/or trace spaces.

We introduce the following convex cone of multipliers on $\Gamma_{C}: \mathbf{M}\left(\mu \lambda_{n}\right)=M_{n} \times M_{t}\left(\mu \lambda_{n}\right)$ where

$$
M_{n}=\left\{\nu \in W_{n}^{\prime}, \nu \geq 0 \text { on } \Gamma_{C}\right\}
$$

and, for any $g \in M_{n}$

$$
M_{t}(g)=\left\{\nu \in W_{t}^{\prime},-g \leq \nu \leq g \text { on } \Gamma_{C}\right\},
$$

and the inequality conditions incorporated in the definitions of $M_{n}$ and $M_{t}(g)$ have to be understood in the dual sense.

We adopt the following notations:

$$
\begin{aligned}
a(\mathbf{u}, \mathbf{v}) & =\int_{\Omega} \boldsymbol{\sigma}(\mathbf{u}): \boldsymbol{\varepsilon}(\mathbf{v}) d \Omega, \quad b(\boldsymbol{\nu}, \mathbf{v})=\left\langle\nu_{n}, v_{n}\right\rangle_{W_{n}^{\prime}, W_{n}}+\left\langle\nu_{t}, v_{t}\right\rangle_{W_{t}^{\prime}, W_{t}}, \\
L(\mathbf{v}) & =\int_{\Omega} \mathbf{f} \cdot \mathbf{v} d \Omega+\int_{\Gamma_{N}} \mathbf{g} \cdot \mathbf{v} d \Gamma
\end{aligned}
$$

for any $\mathbf{u}$ and $\mathbf{v}$ in $\mathbf{V}$ and $\boldsymbol{\nu}$ in $W_{n}^{\prime} \times W_{t}^{\prime}$. In these definitions, the notations $\cdot$ and : represent the canonical inner products in $\mathbb{R}^{2}$ and $\mathcal{S}_{2}$ (the space of second order symmetric tensors on $\mathbb{R}^{2}$ ) respectively and the notation $\langle., .\rangle_{W_{n}^{\prime}, W_{n}}$ represents the duality pairing between $W_{n}^{\prime}$ and $W_{n}$. 
The mixed formulation of the unilateral contact problem with Coulomb friction (2.1)-(2.6) consists then in finding $\mathbf{u} \in \mathbf{V}$ and $\boldsymbol{\lambda} \in \mathbf{M}\left(\mu \lambda_{n}\right)$ such that

$$
\left\{\begin{array}{l}
a(\mathbf{u}, \mathbf{v})+b(\boldsymbol{\lambda}, \mathbf{v})=L(\mathbf{v}), \quad \forall \mathbf{v} \in \mathbf{V}, \\
b(\boldsymbol{\nu}-\boldsymbol{\lambda}, \mathbf{u}) \leq 0, \quad \forall \boldsymbol{\nu}=\left(\nu_{n}, \nu_{t}\right) \in \mathbf{M}\left(\mu \lambda_{n}\right) .
\end{array}\right.
$$

It is easy to see that if $\left(\mathbf{u}, \lambda_{n}, \lambda_{t}\right)$ is a solution of (2.7), then $\lambda_{n}=-\sigma_{n}(\mathbf{u})$ and $\lambda_{t}=-\sigma_{t}(\mathbf{u})$.

The space $W_{n}$ is equipped with the norm

$$
\|w\|_{W_{n}}=\inf _{\mathbf{v} \in \mathbf{V}: v_{n}=w} \text { on } \Gamma_{C}\|\mathbf{v}\|_{1, \Omega}
$$

and a similar holds for $\|\cdot\|_{W_{t}}$. We write afterwards integral terms instead of duality pairings.

Another classical weak formulation of problem (2.1)-(2.6) is a variational inequality: find $\mathbf{u}$ such that

$$
\mathbf{u} \in \mathbf{K}, \quad a(\mathbf{u}, \mathbf{v}-\mathbf{u})-\mu \int_{\Gamma_{C}} \sigma_{n}(\mathbf{u})\left(\left|v_{t}\right|-\left|u_{t}\right|\right) d \Gamma \geq L(\mathbf{v}-\mathbf{u}), \quad \forall \mathbf{v} \in \mathbf{K},
$$

where $\mathbf{K}$ denotes the closed convex cone of admissible displacement fields satisfying the noninterpenetration conditions:

$$
\mathbf{K}=\left\{\mathbf{v} \in \mathbf{V}: \quad v_{n} \leq 0 \text { on } \Gamma_{C}\right\} .
$$

When friction is omitted $(\mu=0)$ then the condition (2.6) simply reduces to $\sigma_{t}(\mathbf{u})=0$ and the frictionless contact problem admits a unique solution according to Stampacchia's theorem (see [37]). The existence of a solution to (2.8) has been proved for small friction coefficients in [43] and the bounds ensuring existence have been improved in [35] and [21]. In [22] existence is stated when $\mu \leq \sqrt{3-4 P} /(2-2 P)$ where $0 \leq P \leq 1 / 2$ denotes Poisson's ratio. In [30,31], some multi-solutions of the problem (2.1)-(2.6) are exhibited for triangular or quadrangular domains. These multiple solutions involve either an infinite set of slipping solutions or two isolated (stick and separation) configurations. Note that these examples of non-uniqueness involve large friction coefficients (i.e. $\mu>\sqrt{(1-P) / P}$ ) and tangential displacements with a constant sign on $\Gamma_{C}$. Actually, it seems that no multi-solution has been detected for an arbitrary small friction coefficient in the continuous case, although such a result exists for finite element approximations in [28], but for a variable geometry. The forthcoming partial uniqueness result is obtained in [46]: it defines some cases where it is possible to affirm that a solution to the Coulomb friction problem is in fact the unique solution. More precisely, if a regular solution to the Coulomb friction problem exists (here the denomination regular means, roughly speaking, that the transition is smooth when the slip direction changes) and if the friction coefficient is small enough then this solution is the only one.

We now introduce the space of multipliers $M$ of the functions $\xi$ defined on $\Gamma_{C}$ such that the following equivalent norm is finite:

$$
\|\xi\|_{M}=\sup _{\substack{v_{t} \in W_{t} \\ v_{t} \neq 0}} \frac{\left\|\xi \cdot v_{t}\right\|_{W_{t}}}{\left\|v_{t}\right\|_{W_{t}}}
$$


Since $\Gamma_{C}$ is assumed to be straight, $M$ contains for any $\varepsilon>0$ the space $H^{1 / 2+\varepsilon}\left(\Gamma_{C}\right)$ (see [41] for a complete discussion on the theory of multipliers in a pair of Hilbert spaces).

The partial uniqueness result is given assuming that $\lambda_{t}=\mu \lambda_{n} \xi$, with $\xi \in M$. It is easy to see that it implies $|\xi| \leq 1$ a.e. on the support of $\lambda_{n}$. More precisely, this implies that $\xi \in \operatorname{Dir}_{t}\left(u_{t}\right)$ a.e. on the support of $\lambda_{n}$, where $\operatorname{Dir}_{t}($.$) is the subdifferential of the convex map x_{t} \longmapsto\left|x_{t}\right|$. This means that it is possible to assume that $\xi \in \operatorname{Dir}_{t}\left(u_{t}\right)$ a.e. on $\Gamma_{C}$.

Proposition 1. Let $\mathbf{u}$ be a solution to Problem (2.8) such that $\lambda_{t}=\mu \lambda_{n} \xi$, with $\xi \in M$, $\xi \in \operatorname{Dir}_{t}\left(u_{t}\right)$ a.e. on $\Gamma_{C}$ and $\mu<\left(C_{0}\|\xi\|_{M}\right)^{-1}$ where $C_{0}$ is independent of $\xi$. Then $\mathbf{u}$ is the unique solution to Problem (2.8).

Proof. see [46]

In two space dimensions $(d=2)$, the case $\xi \equiv 1$ corresponds to an homogeneous sliding direction and the previous result is complementary to the non-uniqueness results obtained in [30, 31].

The multiplier $\xi$ has to vary from -1 to +1 each time the sign of the tangential displacement changes from negative to positive. The set $M$ does not contain any multiplier having a singularity of the first kind. The tangential displacement of the solution $\mathbf{u}$ cannot pass from a negative value to a positive value and being zero only at a single point of $\Gamma_{C}$.

\section{Discretization with the stabilized Lagrange multiplier method}

\subsection{Discrete problem}

Let $\mathbf{V}_{h} \subset \mathbf{V}$ be a family of finite dimensional vector spaces indexed by $h>0$ coming from a regular family $\mathcal{T}_{h}$ (see $[11,13,15]$ ) of triangulations of the domain $\Omega$. For $T \in \mathcal{T}_{h}$, let $h_{T}$ be the diameter of $T$ and $h=\max _{T \in \mathcal{T}_{h}} h_{T}$. We choose standard continuous and piecewise affine functions, i.e.:

$$
\mathbf{V}_{h}=\left\{\mathbf{v}_{h} \in(C(\bar{\Omega}))^{2}: \mathbf{v}_{\left.h\right|_{T}} \in\left(P_{1}(T)\right)^{2}, \forall T \in \mathcal{T}_{h}, \mathbf{v}_{h}=\mathbf{0} \text { on } \Gamma_{D}\right\}
$$

Next, let be given $\mathbf{x}_{0}, \ldots, \mathbf{x}_{N}$ some distinct points lying in $\overline{\Gamma_{C}}$ (note that we do not suppose that these nodes coincide with some nodes of the triangulation $\mathcal{T}_{h}$ ). These nodes form a monodimensional family of meshes of $\Gamma_{C}$ denoted $T_{H}$ and we set $H=\max _{0 \leq i \leq N-1}\left|\mathbf{x}_{i+1}-\mathbf{x}_{i}\right|$. In order to express the contact constraints by using Lagrange multipliers on the contact zone, we have to introduce a finite dimensional space $W_{H}$ approximating $W^{\prime}$. There are two elementary possible choices of $W_{H}$. We can set either

$$
W_{0 H}=\left\{\mu_{H} \in L^{2}\left(\Gamma_{C}\right):\left.\mu_{H}\right|_{\mathbf{x}_{i}, \mathbf{x}_{i+1}[} \in P_{0}(] \mathbf{x}_{i}, \mathbf{x}_{i+1}[), \forall 0 \leq i \leq N-1\right\}
$$


or

$$
W_{1 H}=\left\{\mu_{H} \in C\left(\Gamma_{C}\right):\left.\mu_{H}\right|_{\mathbf{x}_{i}, \mathbf{x}_{i+1}[} \in P_{1}(] \mathbf{x}_{i}, \mathbf{x}_{i+1}[), \forall 0 \leq i \leq N-1\right\} .
$$

The choice of the space $W_{H}$ allows us to define the following closed convex cones:

$$
M_{H n}=\left\{\nu_{H} \in W_{H}: \nu_{H} \geq 0\right\}
$$

and, for $g \in M_{H n}$ :

$$
M_{H t}(g)=\left\{\nu_{H} \in W_{H}:\left|\nu_{H}\right| \leq g\right\} .
$$

The discrete problem is to find $\mathbf{u}_{h} \in \mathbf{V}_{h}$ and $\boldsymbol{\lambda}_{H}=\left(\lambda_{H n}, \lambda_{H t}\right) \in \mathbf{M}_{H}\left(\mu \lambda_{H n}\right)=M_{H n} \times$ $M_{H t}\left(\mu \lambda_{H n}\right)$ such that

$$
\left\{\begin{array}{l}
a\left(\mathbf{u}_{h}, \mathbf{v}_{h}\right)+b\left(\boldsymbol{\lambda}_{H}, \mathbf{v}_{h}\right)+\int_{\Gamma_{C}} \gamma\left(-\lambda_{H n}-\sigma_{n}\left(\mathbf{u}_{h}\right)\right) \sigma_{n}\left(\mathbf{v}_{h}\right) d \Gamma \\
+\int_{\Gamma_{C}} \gamma\left(-\lambda_{H t}-\sigma_{t}\left(\mathbf{u}_{h}\right)\right) \sigma_{t}\left(\mathbf{v}_{h}\right) d \Gamma=L\left(\mathbf{v}_{h}\right), \quad \forall \mathbf{v}_{h} \in \mathbf{V}_{h}, \\
b\left(\boldsymbol{\nu}_{H}-\boldsymbol{\lambda}_{H}, \mathbf{u}_{h}\right)+\int_{\Gamma_{C}} \gamma\left(\nu_{H n}-\lambda_{H n}\right)\left(-\lambda_{H n}-\sigma_{n}\left(\mathbf{u}_{h}\right)\right) d \Gamma \\
+\int_{\Gamma_{C}} \gamma\left(\nu_{H t}-\lambda_{H t}\right)\left(-\lambda_{H t}-\sigma_{t}\left(\mathbf{u}_{h}\right)\right) d \Gamma \leq 0, \quad \forall\left(\nu_{H n}, \nu_{H t}\right) \in \mathbf{M}_{H}\left(\mu \lambda_{H n}\right),
\end{array}\right.
$$

where $\gamma$ is defined constant on each element $T$ as $\gamma=\gamma_{0} h_{T}$ where $\gamma_{0}>0$ is independent of $h$.

Remark 2. The particularity of (3.1) is the presence of stabilization term involving $\gamma$.

Remark 3. When $\gamma=0$, using a fixed point argument, [17] states the existence of a solution and the uniqueness when $\mu \leq C(h)\left(C(h) \sim h^{\frac{1}{2}}\right)$.

The method is consistent in the sense that $\lambda_{H n}$ and $-\sigma_{n}\left(\mathbf{u}_{h}\right)\left(\right.$ resp. $\lambda_{H t}$ and $\left.-\sigma_{t}\left(\mathbf{u}_{h}\right)\right)$ are both some approximations of $\lambda_{n}=-\sigma_{n}(\mathbf{u})$ (resp. $\lambda_{t}=-\sigma_{t}(\mathbf{u})$ ) and the additional stabilization term is vanishing for the solution to the continuous problem. Of course this stabilization term modifies the discrete solution. In fact, it reinforces the correspondence between $\lambda_{H n}$ and $-\sigma_{n}\left(\mathbf{u}_{h}\right)$ (resp. $\lambda_{H t}$ and $\left.-\sigma_{t}\left(\mathbf{u}_{h}\right)\right)$.

\subsection{Existence and uniqueness of the solution}

Proposition 4. For any positive $\mu$ and for $\gamma_{0}$ small enough, problem (3.1) admits at least a solution. 
Proof. Let $\mu>0$ be given. We introduce the problem of friction $P\left(g_{H n}\right)$ with a given threshold $\mu g_{H n}$ and $g_{H n} \in M_{H n}$. It consists of finding $\mathbf{u}_{h} \in \mathbf{V}_{h}$ and $\boldsymbol{\lambda}_{H} \in \mathbf{M}_{H}\left(\mu g_{H n}\right)=M_{H n} \times$ $M_{H t}\left(\mu g_{H n}\right)$ satisfying:

$$
P\left(g_{H n}\right)\left\{\begin{array}{c}
a\left(\mathbf{u}_{h}, \mathbf{v}_{h}\right)+b\left(\boldsymbol{\lambda}_{H}, \mathbf{v}_{h}\right)+\int_{\Gamma_{C}} \gamma\left(-\lambda_{H n}-\sigma_{n}\left(\mathbf{u}_{h}\right)\right) \sigma_{n}\left(\mathbf{v}_{h}\right) d \Gamma \\
+\int_{\Gamma_{C}} \gamma\left(-\lambda_{H t}-\sigma_{t}\left(\mathbf{u}_{h}\right)\right) \sigma_{t}\left(\mathbf{v}_{h}\right) d \Gamma=L\left(\mathbf{v}_{h}\right), \quad \forall \mathbf{v}_{h} \in \mathbf{V}_{h}, \\
b\left(\boldsymbol{\nu}_{H}-\boldsymbol{\lambda}_{H}, \mathbf{u}_{h}\right)+\int_{\Gamma_{C}} \gamma\left(\nu_{H n}-\lambda_{H n}\right)\left(-\lambda_{H n}-\sigma_{n}\left(\mathbf{u}_{h}\right)\right) d \Gamma \\
+\int_{\Gamma_{C}} \gamma\left(\nu_{H t}-\lambda_{H t}\right)\left(-\lambda_{H t}-\sigma_{t}\left(\mathbf{u}_{h}\right)\right) d \Gamma \leq 0, \quad \forall \boldsymbol{\nu}_{H} \in \mathbf{M}_{H}\left(\mu g_{H n}\right) .
\end{array}\right.
$$

Problem (3.2) is equivalent of finding a saddle-point $\left(\mathbf{u}_{h}, \lambda_{H n}, \lambda_{H t}\right)=\left(\mathbf{u}_{h}, \boldsymbol{\lambda}_{H}\right) \in \mathbf{V}_{h} \times$ $\mathbf{M}_{H}\left(\mu g_{H n}\right)$ verifying

$$
\mathcal{L}_{\gamma}\left(\mathbf{u}_{h}, \boldsymbol{\nu}_{H}\right) \leq \mathcal{L}_{\gamma}\left(\mathbf{u}_{h}, \boldsymbol{\lambda}_{H}\right) \leq \mathcal{L}_{\gamma}\left(\mathbf{v}_{h}, \boldsymbol{\lambda}_{H}\right), \quad \forall \mathbf{v}_{h} \in \mathbf{V}_{h}, \forall \boldsymbol{\nu}_{H} \in \mathbf{M}_{H}\left(\mu g_{H n}\right),
$$

where

$\mathcal{L}_{\gamma}\left(\mathbf{v}_{h}, \boldsymbol{\nu}_{H}\right)=\frac{1}{2} a\left(\mathbf{v}_{h}, \mathbf{v}_{h}\right)-L\left(\mathbf{v}_{h}\right)+b\left(\boldsymbol{\nu}_{H}, \mathbf{v}_{h}\right)-\frac{1}{2} \int_{\Gamma_{C}} \gamma\left(\nu_{H n}+\sigma_{n}\left(\mathbf{v}_{h}\right)\right)^{2} d \Gamma-\frac{1}{2} \int_{\Gamma_{C}} \gamma\left(\nu_{H t}+\sigma_{t}\left(\mathbf{v}_{h}\right)\right)^{2} d \Gamma$.

Let $E$ be an edge (of a triangle) on $\Gamma_{C}$ and let $T \in \mathcal{T}_{h}$ be the element containing $E$. Note that we can suppose without loss of generality that $\Gamma_{C}$ is a straight line segment parallel to the $x$-axis and we write $\mathbf{v}=\left(v_{x}, v_{y}\right)$. Consequently we deduce, for any $\mathbf{v}_{h} \in \mathbf{V}_{h}$ :

$$
\begin{aligned}
\left\|\sigma_{n}\left(\mathbf{v}_{h}\right)\right\|_{0, E} & =\left\|\sigma_{y y}\left(\mathbf{v}_{h}\right)\right\|_{0, E}=\frac{|E|^{1 / 2}}{|T|^{1 / 2}}\left\|\sigma_{y y}\left(\mathbf{v}_{h}\right)\right\|_{0, T} \\
& \sim h_{E}^{-1 / 2}\left\|\sigma_{y y}\left(\mathbf{v}_{h}\right)\right\|_{0, T} \sim h_{T}^{-1 / 2}\left\|\sigma_{y y}\left(\mathbf{v}_{h}\right)\right\|_{0, T}
\end{aligned}
$$

Let $h_{\Gamma_{C}}$ be the function equal to $\frac{\gamma}{\gamma_{0}}$ which is independent of $\gamma_{0}$. By summation on all the edges $E \subset \Gamma_{C}$ we get

$$
\left\|h_{\Gamma_{C}}^{1 / 2} \sigma_{n}\left(\mathbf{v}_{h}\right)\right\|_{0, \Gamma_{C}}^{2} \leq C\left\|\sigma_{y y}\left(\mathbf{v}_{h}\right)\right\|_{0, \Omega}^{2} \leq C\left\|\mathbf{v}_{h}\right\|_{1, \Omega}^{2} .
$$

We have the same inequality with $\sigma_{t}\left(\mathbf{v}_{h}\right)$. Hence, from Korn inequality and (3.3), when $\gamma_{0}$ is small enough, there exists $C>0$ such that for any $\mathbf{v}_{h} \in \mathbf{V}_{h}$ :

$$
a\left(\mathbf{v}_{h}, \mathbf{v}_{h}\right)-\int_{\Gamma_{C}} \gamma\left(\sigma_{n}\left(\mathbf{v}_{h}\right)\right)^{2} d \Gamma-\int_{\Gamma_{C}} \gamma\left(\sigma_{t}\left(\mathbf{v}_{h}\right)\right)^{2} d \Gamma \geq C\left\|\mathbf{v}_{h}\right\|_{1, \Omega}^{2}
$$

By using classical arguments on saddle-point problems as Haslinger, Hlaváček and Nečas ([26], p.338), we deduce that there exists such a saddle-point. Indeed, the existence of a solution to problem (3.2) when $\gamma_{0}$ is small enough follows from the fact that 
- $\mathbf{V}_{h}$ and $\mathbf{M}_{H}\left(\mu g_{H n}\right)$ are two nonempty closed convex sets,

- $\mathcal{L}_{\gamma}(.,$.$) is continuous on \mathbf{V}_{h} \times W_{H}^{2}$,

- $\mathcal{L}_{\gamma}\left(\mathbf{v}_{h},.\right)$ (resp. $\mathcal{L}_{\gamma}\left(., \boldsymbol{\nu}_{H}\right)$ ) is strictly concave (resp. strictly convex) for any $\mathbf{v}_{h} \in \mathbf{V}_{h}$ (resp. for any $\boldsymbol{\nu}_{H} \in \mathbf{M}_{H}\left(\mu g_{H n}\right)$ ),

- by taking $\boldsymbol{\nu}_{H}=0$ and by (3.4) with $\gamma_{0}$ small enough, we obtain

$$
\mathcal{L}_{\gamma}\left(\mathbf{v}_{h}, 0\right) \geq \frac{C}{2}\left\|\mathbf{v}_{h}\right\|_{1, \Omega}^{2}-\|L\|_{V}\left\|\mathbf{v}_{h}\right\|_{1, \Omega}
$$

which tends to infinity when $\left\|\mathbf{v}_{h}\right\|_{1, \Omega} \longrightarrow \infty$. So $\lim _{\mathbf{v}_{h} \in \mathbf{V}_{h},\left\|\mathbf{v}_{h}\right\|_{1, \Omega} \rightarrow \infty} \mathcal{L}_{\gamma}\left(\mathbf{v}_{h}, 0\right)=+\infty$

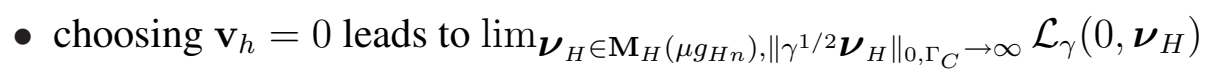

$$
=-\frac{1}{2} \int_{\Gamma_{C}} \gamma \nu_{H n}^{2} d \Gamma-\frac{1}{2} \int_{\Gamma_{C}} \gamma \nu_{H t}^{2} d \Gamma=-\infty .
$$

The strict convexity of $a(.,$.$) implies that the first argument \mathbf{u}_{h}$ is unique.

Besides, suppose that the second argument is not unique. The inequality in (3.2) allow us to write by choosing $\nu_{H n}=\lambda_{H n}^{2}$ and $\nu_{H t}=\lambda_{H t}^{2}$,

$$
\begin{array}{r}
\int_{\Gamma_{C}}\left(\lambda_{H n}^{2}-\lambda_{H n}^{1}\right) u_{h n} d \Gamma+\int_{\Gamma_{C}} \gamma\left(\lambda_{H n}^{2}-\lambda_{H n}^{1}\right)\left(-\lambda_{H n}^{1}-\sigma_{n}\left(\mathbf{u}_{h}\right)\right) d \Gamma \\
+\int_{\Gamma_{C}}\left(\lambda_{H t}^{2}-\lambda_{H t}^{1}\right) u_{h t} d \Gamma+\int_{\Gamma_{C}} \gamma\left(\lambda_{H t}^{2}-\lambda_{H t}^{1}\right)\left(-\lambda_{H t}^{1}-\sigma_{t}\left(\mathbf{u}_{h}\right)\right) d \Gamma \leq 0
\end{array}
$$

and by choosing $\nu_{H n}=\lambda_{H n}^{1}$ and $\nu_{H t}=\lambda_{H t}^{1}$,

$$
\begin{array}{r}
\int_{\Gamma_{C}}\left(\lambda_{H n}^{1}-\lambda_{H n}^{2}\right) u_{h n} d \Gamma+\int_{\Gamma_{C}} \gamma\left(\lambda_{H n}^{1}-\lambda_{H n}^{2}\right)\left(-\lambda_{H n}^{2}-\sigma_{n}\left(\mathbf{u}_{h}\right)\right) d \Gamma \\
+\int_{\Gamma_{C}}\left(\lambda_{H t}^{1}-\lambda_{H t}^{2}\right) u_{h t} d \Gamma+\int_{\Gamma_{C}} \gamma\left(\lambda_{H t}^{1}-\lambda_{H t}^{2}\right)\left(-\lambda_{H t}^{2}-\sigma_{t}\left(\mathbf{u}_{h}\right)\right) d \Gamma \leq 0
\end{array}
$$

By addition we obtain $-\left\|\gamma^{1 / 2}\left(\lambda_{H n}^{1}-\lambda_{H n}^{2}\right)\right\|_{0, \Gamma_{C}}^{2}-\left\|\gamma^{1 / 2}\left(\lambda_{H t}^{1}-\lambda_{H t}^{2}\right)\right\|_{0, \Gamma_{C}}^{2} \geq 0$ and we come to the conclusion that $\lambda_{H n}^{1}=\lambda_{H n}^{2}, \lambda_{H t}^{1}=\lambda_{H t}^{2}$. Consequently, the second argument $\lambda_{H}$ is unique and (3.2) admits a unique solution. The next definition is a straightforward consequence of the definition of problems (3.1) and (3.2).

Definition 1. The solutions of Coulomb's discrete frictional contact problem (3.1) are the solutions of $P\left(\lambda_{H n}\right)$ where $\lambda_{H n}$ is a fixed point of $\Phi_{H}$ defined as follows:

$$
\begin{aligned}
\Phi_{H}: M_{H n} & \longrightarrow M_{H n} \\
g_{H n} & \longmapsto \lambda_{H n} .
\end{aligned}
$$

where $\left(\mathbf{u}_{h}, \boldsymbol{\lambda}_{H}\right)$ is the solution of $P\left(g_{H n}\right)$. 
Continuation of the proof: To establish existence of a fixed point of $\Phi_{H}$, we use Brouwer's fixed point theorem.

Step 1 . We prove that the mapping $\Phi_{H}$ is continuous.

Let $\left(\mathbf{u}_{h}, \lambda_{H n}, \lambda_{H t}\right)$ and $\left(\overline{\mathbf{u}_{h}}, \overline{\lambda_{H n}}, \overline{\lambda_{H t}}\right)$ be the solutions of $\left(P\left(g_{H n}\right)\right)$ and $\left(P\left(\overline{g_{H n}}\right)\right)$ respectively. On the one hand, we get

$$
\left\|\gamma^{1 / 2}\left(\lambda_{H n}-\overline{\lambda_{H n}}\right)\right\|_{0, \Gamma_{C}}^{2}=\int_{\Gamma_{C}} \gamma \lambda_{H n}^{2} d \Gamma-2 \int_{\Gamma_{C}} \gamma \lambda_{H n} \overline{\lambda_{H n}} d \Gamma+\int_{\Gamma_{C}} \gamma{\overline{\lambda_{H n}}}^{2} d \Gamma
$$

By using (3.2) and $\nu_{H t}=\lambda_{H t}$, we obtain $\forall \nu_{H n} \in M_{H n}$

$$
\begin{array}{r}
\int_{\Gamma_{C}}\left(\nu_{H n}-\lambda_{H n}\right) u_{h n} d \Gamma+\int_{\Gamma_{C}} \gamma\left(\nu_{H n}-\lambda_{H n}\right)\left(-\lambda_{H n}-\sigma_{n}\left(\mathbf{u}_{h}\right)\right) d \Gamma \leq 0 \\
\int_{\Gamma_{C}} \gamma \lambda_{H n}^{2} d \Gamma \leq \int_{\Gamma_{C}}\left(\lambda_{H n}-\nu_{H n}\right) u_{h n} d \Gamma+\int_{\Gamma_{C}} \gamma\left(\nu_{H n}-\lambda_{H n}\right) \sigma_{n}\left(\mathbf{u}_{h}\right) d \Gamma+\int_{\Gamma_{C}} \gamma \nu_{H n} \lambda_{H n} d \Gamma
\end{array}
$$

and

$$
\int_{\Gamma_{C}} \gamma{\overline{\lambda_{H n}}}^{2} d \Gamma \leq \int_{\Gamma_{C}}\left(\overline{\lambda_{H n}}-\nu_{H n}\right) \overline{u_{h n}} d \Gamma+\int_{\Gamma_{C}} \gamma\left(\nu_{H n}-\overline{\lambda_{H n}}\right) \sigma_{n}\left(\overline{\mathbf{u}_{h}}\right) d \Gamma+\int_{\Gamma_{C}} \gamma \nu_{H n} \overline{\lambda_{H n}} d \Gamma .
$$

Taking $\nu_{H n}=\overline{\lambda_{H n}}$ in (3.5) and $\nu_{H n}=\lambda_{H n}$ in (3.6) and (3.3) implies:

$$
\begin{aligned}
\left\|\gamma^{1 / 2}\left(\lambda_{H n}-\overline{\lambda_{H n}}\right)\right\|_{0, \Gamma_{C} \leq}^{2} \leq & \int_{\Gamma_{C}}\left(\lambda_{H n}-\overline{\lambda_{H n}}\right) u_{h n} d \Gamma+\int_{\Gamma_{C}} \gamma\left(\overline{\lambda_{H n}}-\lambda_{H n}\right) \sigma_{n}\left(\mathbf{u}_{h}\right) d \Gamma \\
& +\int_{\Gamma_{C}}\left(\overline{\lambda_{H n}}-\lambda_{H n}\right) \overline{u_{h n}} d \Gamma+\int_{\Gamma_{C}} \gamma\left(\lambda_{H n}-\overline{\lambda_{H n}}\right) \sigma_{n}\left(\overline{\mathbf{u}_{h}}\right) d \Gamma \\
\leq & \left\|\gamma^{1 / 2}\left(\lambda_{H n}-\overline{\lambda_{H n}}\right)\right\|_{0, \Gamma_{C}} \gamma_{0}^{-1 / 2}\left\|h_{\Gamma_{C}}^{-1 / 2}\left(u_{h n}-\overline{u_{h n}}\right)\right\|_{0, \Gamma_{C}} \\
& +\left\|\gamma^{1 / 2}\left(\lambda_{H n}-\overline{\lambda_{H n}}\right)\right\|_{0, \Gamma_{C}} \gamma_{0}^{1 / 2}\left\|h_{\Gamma_{C}}^{1 / 2}\left(\sigma_{n}\left(\mathbf{u}_{h}\right)-\sigma_{n}\left(\overline{\mathbf{u}_{h}}\right)\right)\right\|_{0, \Gamma_{C}} \\
\lesssim & \left\|\gamma^{1 / 2}\left(\lambda_{H n}-\overline{\lambda_{H n}}\right)\right\|_{0, \Gamma_{C}} \gamma_{0}^{-1 / 2}\left\|\mathbf{u}_{h}-\overline{\mathbf{u}_{h}}\right\|_{1, \Omega} \\
& +\left\|\gamma^{1 / 2}\left(\lambda_{H n}-\overline{\lambda_{H n}}\right)\right\|_{0, \Gamma_{C}} \gamma_{0}^{1 / 2}\left\|\mathbf{u}_{h}-\overline{\mathbf{u}_{h}}\right\|_{1, \Omega}
\end{aligned}
$$

Hence, we get a first estimate

$$
\left\|\gamma^{1 / 2}\left(\lambda_{H n}-\overline{\lambda_{H n}}\right)\right\|_{0, \Gamma_{C}} \lesssim\left(\gamma_{0}^{1 / 2}+\gamma_{0}^{-1 / 2}\right)\left\|\mathbf{u}_{h}-\overline{\mathbf{u}_{h}}\right\|_{1, \Omega}
$$

On the other hand, we have from (3.2) $\forall \mathbf{v}_{h} \in \mathbf{V}_{h}$,

$$
\begin{gathered}
a\left(\mathbf{u}_{h}, \mathbf{v}_{h}\right)+\int_{\Gamma_{C}} \lambda_{H n} v_{h n} d \Gamma+\int_{\Gamma_{C}} \gamma\left(-\lambda_{H n}-\sigma_{n}\left(\mathbf{u}_{h}\right)\right) \sigma_{n}\left(\mathbf{v}_{h}\right) d \Gamma \\
\quad+\int_{\Gamma_{C}} \lambda_{H t} v_{h t} d \Gamma+\int_{\Gamma_{C}} \gamma\left(-\lambda_{H t}-\sigma_{t}\left(\mathbf{u}_{h}\right)\right) \sigma_{t}\left(\mathbf{v}_{h}\right) d \Gamma=L\left(\mathbf{v}_{h}\right)
\end{gathered}
$$


and

$$
\begin{gathered}
a\left(\overline{\mathbf{u}_{h}}, \mathbf{v}_{h}\right)+\int_{\Gamma_{C}} \overline{\lambda_{H n}} v_{h n} d \Gamma+\int_{\Gamma_{C}} \gamma\left(-\overline{\lambda_{H n}}-\sigma_{n}\left(\overline{\mathbf{u}_{h}}\right)\right) \sigma_{n}\left(\mathbf{v}_{h}\right) d \Gamma \\
\quad+\int_{\Gamma_{C}} \overline{\lambda_{H t}} v_{h t} d \Gamma+\int_{\Gamma_{C}} \gamma\left(-\overline{\lambda_{H t}}-\sigma_{t}\left(\overline{\mathbf{u}_{h}}\right)\right) \sigma_{t}\left(\mathbf{v}_{h}\right) d \Gamma=L\left(\mathbf{v}_{h}\right)
\end{gathered}
$$

Choosing $\mathbf{v}_{h}=\mathbf{u}_{h}-\overline{\mathbf{u}}_{h}$ in (3.8) and $\mathbf{v}_{h}=\overline{\mathbf{u}_{h}}-\mathbf{u}_{h}$ in (3.9) implies by addition:

$$
\begin{aligned}
a\left(\mathbf{u}_{h}-\overline{\mathbf{u}_{h}}, \mathbf{u}_{h}-\overline{\mathbf{u}_{h}}\right)= & \int_{\Gamma_{C}}\left(\overline{\lambda_{H n}}-\lambda_{H n}\right)\left(u_{h n}-\overline{u_{h n}}\right) d \Gamma+\int_{\Gamma_{C}}\left(\overline{\lambda_{H t}}-\lambda_{H t}\right)\left(u_{h t}-\overline{u_{h t}}\right) d \Gamma \\
& +\int_{\Gamma_{C}} \gamma\left(\overline{\lambda_{H n}}-\lambda_{H n}-\sigma_{n}\left(\mathbf{u}_{h}\right)+\sigma_{n}\left(\overline{\mathbf{u}_{h}}\right)\right) \sigma_{n}\left(\overline{\mathbf{u}_{h}}-\mathbf{u}_{h}\right) d \Gamma \\
& +\int_{\Gamma_{C}} \gamma\left(\overline{\lambda_{H t}}-\lambda_{H t}-\sigma_{t}\left(\mathbf{u}_{h}\right)+\sigma_{t}\left(\overline{\mathbf{u}_{h}}\right)\right) \sigma_{t}\left(\overline{\mathbf{u}_{h}}-\mathbf{u}_{h}\right) d \Gamma
\end{aligned}
$$

Let us notice that the inequality in (3.2) is obviously equivalent to the two following conditions:

$$
\begin{gathered}
\int_{\Gamma_{C}}\left(\nu_{H n}-\lambda_{H n}\right) u_{h n} d \Gamma+\int_{\Gamma_{C}} \gamma\left(-\lambda_{H n}-\sigma_{n}\left(\mathbf{u}_{h}\right)\right)\left(\nu_{H n}-\lambda_{H n}\right) d \Gamma \leq 0, \forall \nu_{H n} \in M_{H n},(3.1) \\
\int_{\Gamma_{C}}\left(\nu_{H t}-\lambda_{H t}\right) u_{h t} d \Gamma+\int_{\Gamma_{C}} \gamma\left(-\lambda_{H t}-\sigma_{t}\left(\mathbf{u}_{h}\right)\right)\left(\nu_{H t}-\lambda_{H t}\right) d \Gamma \leq 0 \\
\forall \nu_{H t} \in M_{H t}\left(\mu g_{H n}\right) .
\end{gathered}
$$

According to the definition of $M_{H n}$, we can choose $\nu_{H n}=0$ and $\nu_{H n}=2 \lambda_{H n}$ in (3.11) which gives $\forall \nu_{H n} \in M_{H n}$

$\int_{\Gamma_{C}} \lambda_{H n} u_{h n} d \Gamma+\int_{\Gamma_{C}} \gamma\left(-\lambda_{H n}-\sigma_{n}\left(\mathbf{u}_{h}\right)\right) \lambda_{H n} d \Gamma=0$ and $\int_{\Gamma_{C}} \nu_{H n} u_{h n} d \Gamma+\int_{\Gamma_{C}} \gamma\left(-\lambda_{H n}-\sigma_{n}\left(\mathbf{u}_{h}\right)\right) \nu_{H n} d \Gamma \leq 0$,

from which we deduce that

$$
\begin{gathered}
-\int_{\Gamma_{C}} \lambda_{H n} u_{h n} d \Gamma+\int_{\Gamma_{C}} \gamma \lambda_{H n} \sigma_{n}\left(\mathbf{u}_{h}\right) d \Gamma=-\int_{\Gamma_{C}} \gamma \lambda_{H n}^{2} d \Gamma, \\
\int_{\Gamma_{C}} \overline{\lambda_{H n}} u_{h n} d \Gamma-\int_{\Gamma_{C}} \gamma \overline{\lambda_{H n}} \sigma_{n}\left(\mathbf{u}_{h}\right) d \Gamma \leq \int_{\Gamma_{C}} \gamma \overline{\lambda_{H n}} \lambda_{H n} d \Gamma .
\end{gathered}
$$

Similarly we have

$$
\begin{gathered}
-\int_{\Gamma_{C}} \overline{\lambda_{H n}} \overline{u_{h n}} d \Gamma+\int_{\Gamma_{C}} \gamma \overline{\lambda_{H n}} \sigma_{n}\left(\overline{\mathbf{u}_{h}}\right) d \Gamma=-\int_{\Gamma_{C}} \gamma{\overline{\lambda_{H n}}}^{2} d \Gamma \\
\int_{\Gamma_{C}} \lambda_{H n} \overline{u_{h n}} d \Gamma-\int_{\Gamma_{C}} \gamma \lambda_{H n} \sigma_{n}\left(\overline{\mathbf{u}_{h}}\right) d \Gamma \leq \int_{\Gamma_{C}} \gamma \overline{\lambda_{H n}} \lambda_{H n} d \Gamma .
\end{gathered}
$$


Denoting by $\alpha$ the ellipticity constant of the bilinear form $a(.,),.(3.10)$ becomes

$$
\begin{aligned}
\alpha\left\|\mathbf{u}_{h}-\overline{\mathbf{u}_{h}}\right\|_{1, \Omega}^{2} \leq & -\int_{\Gamma_{C}} \gamma{\overline{\lambda_{H n}}}^{2} d \Gamma+2 \int_{\Gamma_{C}} \gamma \overline{\lambda_{H n}} \lambda_{H n} d \Gamma-\int_{\Gamma_{C}} \gamma \lambda_{H n}^{2} d \Gamma \\
& +\int_{\Gamma_{C}} \gamma \sigma_{n}\left(\overline{\mathbf{u}_{h}}-\mathbf{u}_{h}\right)^{2} d \Gamma+\int_{\Gamma_{C}}\left(\overline{\lambda_{H t}}-\lambda_{H t}\right)\left(u_{h t}-\overline{u_{h t}}\right) d \Gamma \\
& +\int_{\Gamma_{C}} \gamma\left(\overline{\lambda_{H t}}-\lambda_{H t}-\sigma_{t}\left(\mathbf{u}_{h}\right)+\sigma_{t}\left(\overline{\mathbf{u}_{h}}\right)\right) \sigma_{t}\left(\overline{\mathbf{u}_{h}}-\mathbf{u}_{h}\right) d \Gamma \\
= & -\int_{\Gamma_{C}} \gamma\left(\overline{\lambda_{H n}}-\lambda_{H n}\right)^{2} d \Gamma+\gamma_{0}\left\|h_{\Gamma_{C}}^{1 / 2} \sigma_{n}\left(\mathbf{u}_{h}-\overline{\mathbf{u}_{h}}\right)\right\|_{0, \Gamma_{C}}^{2}+\int_{\Gamma_{C}}\left(\overline{\lambda_{H t}}-\lambda_{H t}\right)\left(u_{h t}-\overline{u_{h t}}\right) d \Gamma \\
& +\int_{\Gamma_{C}} \gamma\left(\overline{\lambda_{H t}}-\lambda_{H t}-\sigma_{t}\left(\mathbf{u}_{h}\right)+\sigma_{t}\left(\overline{\mathbf{u}_{h}}\right)\right) \sigma_{t}\left(\overline{\mathbf{u}_{h}}-\mathbf{u}_{h}\right) d \Gamma \\
\leq & C \gamma_{0}\left\|\mathbf{u}_{h}-\overline{\mathbf{u}_{h}}\right\|_{1, \Omega}^{2}+\int_{\Gamma_{C}}\left(\overline{\lambda_{H t}}-\lambda_{H t}\right)\left(u_{h t}-\overline{u_{h t}}\right) d \Gamma \\
& +\int_{\Gamma_{C}} \gamma\left(\overline{\lambda_{H t}}-\lambda_{H t}-\sigma_{t}\left(\mathbf{u}_{h}\right)+\sigma_{t}\left(\overline{\mathbf{u}_{h}}\right)\right) \sigma_{t}\left(\overline{\mathbf{u}_{h}}-\mathbf{u}_{h}\right) d \Gamma
\end{aligned}
$$

Moreover from (3.12), (3.3), $\forall \nu_{H t} \in M_{H t}\left(\mu g_{H n}\right)$ and $\forall \overline{\nu_{H t}} \in M_{H t}\left(\mu \overline{g_{H n}}\right)$ :

$$
\begin{aligned}
& \int_{\Gamma_{C}}\left(\overline{\lambda_{H t}}-\lambda_{H t}\right)\left(u_{h t}-\overline{u_{h t}}\right) d \Gamma+\int_{\Gamma_{C}} \gamma\left(\overline{\lambda_{H t}}-\lambda_{H t}-\sigma_{t}\left(\mathbf{u}_{h}\right)+\sigma_{t}\left(\overline{\mathbf{u}_{h}}\right)\right) \sigma_{t}\left(\overline{\mathbf{u}_{h}}-\mathbf{u}_{h}\right) d \Gamma \\
& =\int_{\Gamma_{C}} \overline{\lambda_{H t}} u_{h t} d \Gamma+\int_{\Gamma_{C}} \lambda_{H t} \overline{u_{h t}} d \Gamma-\int_{\Gamma_{C}} \gamma \overline{\lambda_{H t}}\left(\lambda_{H t}+\sigma_{t}\left(\mathbf{u}_{h}\right)\right) d \Gamma \\
& -\int_{\Gamma_{C}} \gamma \lambda_{H t}\left(\overline{\lambda_{H t}}+\sigma_{t}\left(\overline{\mathbf{u}_{h}}\right)\right) d \Gamma+2 \int_{\Gamma_{C}} \gamma \overline{\lambda_{H t}} \lambda_{H t} d \Gamma+\int_{\Gamma_{C}} \gamma \sigma_{t}\left(\overline{\mathbf{u}_{h}}-\mathbf{u}_{h}\right)^{2} d \Gamma \\
& -\int_{\Gamma_{C}} \lambda_{H t} u_{h t} d \Gamma+\int_{\Gamma_{C}} \gamma \lambda_{H t} \sigma_{t}\left(\mathbf{u}_{h}\right) d \Gamma-\int_{\Gamma_{C}} \overline{\lambda_{H t}} \overline{u_{h t}} d \Gamma+\int_{\Gamma_{C}} \gamma \overline{\lambda_{H t}} \sigma_{t}\left(\overline{\mathbf{u}_{h}}\right) d \Gamma \\
& \leq \int_{\Gamma_{C}} \overline{\lambda_{H t}} u_{h t} d \Gamma+\int_{\Gamma_{C}} \lambda_{H t} \overline{u_{h t}} d \Gamma-\int_{\Gamma_{C}} \gamma \overline{\lambda_{H t}}\left(\lambda_{H t}+\sigma_{t}\left(\mathbf{u}_{h}\right)\right) d \Gamma \\
& -\int_{\Gamma_{C}} \gamma \lambda_{H t}\left(\overline{\lambda_{H t}}+\sigma_{t}\left(\overline{\mathbf{u}_{h}}\right)\right) d \Gamma+2 \int_{\Gamma_{C}} \gamma \overline{\lambda_{H t}} \lambda_{H t} d \Gamma+\gamma_{0}\left\|h_{\Gamma_{C}}^{1 / 2} \sigma_{t}\left(\mathbf{u}_{h}-\overline{\mathbf{u}_{h}}\right)\right\|_{0, \Gamma_{C}}^{2} \\
& -\int_{\Gamma_{C}} \nu_{H t} u_{h t} d \Gamma+\int_{\Gamma_{C}} \gamma \nu_{H t}\left(\lambda_{H t}+\sigma_{t}\left(\mathbf{u}_{h}\right)\right) d \Gamma-\int_{\Gamma_{C}} \gamma \lambda_{H t}^{2} d \Gamma \\
& -\int_{\Gamma_{C}} \overline{\nu_{H t} u_{h t}} d \Gamma+\int_{\Gamma_{C}} \gamma \overline{\nu_{H t}}\left(\overline{\lambda_{H t}}+\sigma_{t}\left(\overline{\mathbf{u}_{h}}\right)\right) d \Gamma-\int_{\Gamma_{C}} \gamma{\overline{\lambda_{H t}}}^{2} d \Gamma
\end{aligned}
$$




$$
\begin{aligned}
\leq & -\int_{\Gamma_{C}} \nu_{H t}\left(u_{h t}-\gamma \lambda_{H t}-\gamma \sigma_{t}\left(\mathbf{u}_{h}\right)\right) d \Gamma-\int_{\Gamma_{C}} \overline{\overline{\nu_{H}}}\left(\overline{u_{h t}}-\gamma \overline{\lambda_{H t}}-\gamma \sigma_{t}\left(\overline{\mathbf{u}_{h}}\right)\right) d \Gamma \\
& +\int_{\Gamma_{C}} \lambda_{H t}\left(\overline{u_{h t}}-\gamma \overline{\lambda_{H t}}-\gamma \sigma_{t}\left(\overline{\mathbf{u}_{h}}\right)\right) d \Gamma+\int_{\Gamma_{C}} \overline{\lambda_{H t}}\left(u_{h t}-\gamma \lambda_{H t}-\gamma \sigma_{t}\left(\mathbf{u}_{h}\right)\right) d \Gamma \\
& -\int_{\Gamma_{C}} \gamma\left(\overline{\lambda_{H t}}-\lambda_{H t}\right)^{2} d \Gamma+C \gamma_{0}\left\|\mathbf{u}_{h}-\overline{\mathbf{u}_{h}}\right\|_{1, \Omega}^{2}
\end{aligned}
$$

To evaluate the latter inequality, let us first introduce the matrices $M_{1}=\left(m 1_{i j}\right)_{1 \leq i, j \leq p}, M_{2}=$ $\left(m 2_{i j}\right)_{1 \leq i \leq p, 1 \leq j \leq q}, M_{3}=\left(m 3_{i j}\right)_{1 \leq i \leq p, 1 \leq j \leq r}$ on $\Gamma_{C}$ as

$$
m 1_{i j}=\int_{\Gamma_{C}} \phi_{i} \phi_{j} d \Gamma, m 2_{i j}=\int_{\Gamma_{C}} \phi_{i} \psi_{j} d \Gamma, m 3_{i j}=\int_{\Gamma_{C}} \phi_{i} \chi_{j} d \Gamma
$$

where $\phi_{i}$ and $\chi_{i}$ are the basis functions on $\mathbf{V}_{\left.h\right|_{\Gamma_{C}}} . n$ and $\psi_{i}$ are the basis functions on $W_{H}$.

Let $U_{T}, \overline{U_{T}}, \Lambda_{T}, \overline{\Lambda_{T}}, \Sigma_{T}, \overline{\Sigma_{T}}, G_{N}, \overline{G_{N}}$ denote the vectors of components the element values of $u_{h t}, \overline{u_{h t}}, \lambda_{H t}, \overline{\lambda_{H t}}, \sigma_{t}\left(\mathbf{u}_{h}\right), \sigma_{t}\left(\overline{\mathbf{u}_{h}}\right), g_{H n}$ and $\overline{g_{H n}}$ respectively. From the definition of $M_{H t}\left(\mu g_{H n}\right)$, we get $\forall N \in \mathbb{R}^{p}$ such that $\left|N_{i}\right| \leq \mu\left(G_{N}\right)_{i}, 1 \leq i \leq p$

$$
\begin{aligned}
& -\int_{\Gamma_{C}} \nu_{H t}\left(u_{h t}-\gamma \lambda_{H t}-\gamma \sigma_{t}\left(\mathbf{u}_{h}\right)\right) d \Gamma \\
& =-\int_{\Gamma_{G=1}}^{p} \nu_{H t}\left(x_{i}\right) \phi_{i}\left(\sum_{j=1}^{p} u_{h t}\left(x_{j}\right) \phi_{j}-\sum_{j=1}^{q} \gamma \lambda_{H t}\left(x_{j}\right) \psi_{j}-\sum_{j=1}^{r} \gamma \sigma_{t}\left(\mathbf{u}_{h}\right)\left(x_{j}\right) \chi_{j}\right) d \Gamma \\
& =-\sum_{i=1}^{p} N_{i}\left(\left(M_{1} U_{T}\right)_{i}-\gamma\left(M_{2} \Lambda_{T}\right)_{i}-\gamma\left(M_{3} \Sigma_{T}\right)_{i}\right) .
\end{aligned}
$$

It is easy to construct a vector $\mathrm{N}$ minimizing the sum : if $\left(M_{1} U_{T}\right)_{i}-\gamma\left(M_{2} \Lambda_{T}\right)_{i}-\gamma\left(M_{3} \Sigma_{T}\right)_{i} \geq 0$, we choose $N_{i}=\mu\left(G_{N}\right)_{i}$ and if $\left(M_{1} U_{T}\right)_{i}-\gamma\left(M_{2} \Lambda_{T}\right)_{i}-\gamma\left(M_{3} \Sigma_{T}\right)_{i} \leq 0$, we choose $N_{i}=-\mu\left(G_{N}\right)_{i}$ and it yields the following bound:

$$
-\int_{\Gamma_{C}} \nu_{H t}\left(u_{h t}-\gamma \lambda_{H t}-\gamma \sigma_{t}\left(\mathbf{u}_{h}\right)\right) d \Gamma=-\sum_{i=1}^{p} \mu\left(G_{N}\right)_{i}\left|\left(M_{1} U_{T}\right)_{i}-\gamma\left(M_{2} \Lambda_{T}\right)_{i}-\gamma\left(M_{3} \Sigma_{T}\right)_{i}\right| .
$$

A similar expression can be obtained when integrating the term $-\int_{\Gamma_{C}} \overline{\overline{\nu_{H}}}\left(\overline{u_{h t}}-\gamma \overline{\lambda_{H t}}-\gamma \sigma_{t}\left(\overline{\mathbf{u}_{h}}\right)\right) d \Gamma$. Besides from the definition of $M_{H t}\left(\mu g_{H n}\right)$, we get:

$$
\begin{aligned}
\int_{\Gamma_{C}} \lambda_{H t}\left(\overline{u_{h t}}-\gamma \overline{\lambda_{H t}}-\gamma \sigma_{t}\left(\overline{\mathbf{u}_{h}}\right)\right) d \Gamma & =\sum_{i=1}^{p}\left(\Lambda_{T}\right)_{i}\left(\left(M_{1} \overline{U_{T}}\right)_{i}-\gamma\left(M_{2} \overline{\Lambda_{T}}\right)_{i}-\gamma\left(M_{3} \overline{\Sigma_{T}}\right)_{i}\right) \\
& \leq \sum_{i=1}^{p}\left|\left(\Lambda_{T}\right)_{i}\right|\left|\left(M_{1} \overline{U_{T}}\right)_{i}-\gamma\left(M_{2} \overline{\Lambda_{T}}\right)_{i}-\gamma\left(M_{3} \overline{\Sigma_{T}}\right)_{i}\right| \\
& \leq \sum_{i=1}^{p} \mu\left(G_{N}\right)_{i}\left|\left(M_{1} \overline{U_{T}}\right)_{i}-\gamma\left(M_{2} \overline{\Lambda_{T}}\right)_{i}-\gamma\left(M_{3} \overline{\Sigma_{T}}\right)_{i}\right|
\end{aligned}
$$


A similar expression can be obtained when integrating the term $\int_{\Gamma_{C}} \overline{\lambda_{H t}}\left(u_{h t}-\gamma \lambda_{H t}-\gamma \sigma_{t}\left(\mathbf{u}_{h}\right)\right) d \Gamma$.

Finally, (3.14) becomes with the Hölder inequality:

$$
\begin{aligned}
& \int_{\Gamma_{C}}\left(\overline{\lambda_{H t}}-\lambda_{H t}\right)\left(u_{h t}-\overline{u_{h t}}\right) d \Gamma+\int_{\Gamma_{C}} \gamma\left(\overline{\lambda_{H t}}-\lambda_{H t}-\sigma_{t}\left(\mathbf{u}_{h}\right)+\sigma_{t}\left(\overline{\mathbf{u}_{h}}\right)\right) \sigma_{t}\left(\overline{\mathbf{u}_{h}}-\mathbf{u}_{h}\right) d \Gamma \\
\lesssim & \mu \sum_{i=1}^{p}\left(G_{N}-\overline{G_{N}}\right)_{i}\left(\left|\left(M_{1} \overline{U_{T}}\right)_{i}-\gamma\left(M_{2} \overline{\Lambda_{T}}\right)_{i}-\gamma\left(M_{3} \overline{\Sigma_{T}}\right)_{i}\right|-\left|\left(M_{1} U_{T}\right)_{i}-\gamma\left(M_{2} \Lambda_{T}\right)_{i}-\gamma\left(M_{3} \Sigma_{T}\right)_{i}\right|\right) \\
& +C \gamma_{0}\left\|\mathbf{u}_{h}-\overline{\mathbf{u}_{h}}\right\|_{1, \Omega}^{2}-\int_{\Gamma_{C}} \gamma\left(\overline{\lambda_{H t}}-\lambda_{H t}\right)^{2} d \Gamma \\
\lesssim & \mu \sum_{i=1}^{p}\left(G_{N}-\overline{G_{N}}\right)_{i}\left|\left(M_{1} \overline{U_{T}}\right)_{i}-\gamma\left(M_{2} \overline{\Lambda_{T}}\right)_{i}-\gamma\left(M_{3} \overline{\Sigma_{T}}\right)_{i}-\left(M_{1} U_{T}\right)_{i}+\gamma\left(M_{2} \Lambda_{T}\right)_{i}+\gamma\left(M_{3} \Sigma_{T}\right)_{i}\right| \\
& +C \gamma_{0}\left\|\mathbf{u}_{h}-\overline{\mathbf{u}_{h}}\right\|_{1, \Omega}^{2}-\int_{\Gamma_{C}} \gamma\left(\overline{\lambda_{H t}}-\lambda_{H t}\right)^{2} d \Gamma \\
\lesssim & \mu\left(\sum_{i=1}^{p}\left(G_{N}-\overline{G_{N}}\right)_{i}^{2}\right)^{\frac{1}{2}}\left(\left(\sum_{i=1}^{p}\left(M_{1}\left(U_{T}-\overline{U_{T}}\right)\right)_{i}^{2}\right)^{\frac{1}{2}}+\left(\sum_{i=1}^{p}\left(\gamma M_{2}\left(\Lambda_{T}-\overline{\Lambda_{T}}\right)\right)_{i}^{2}\right)^{\frac{1}{2}}\right. \\
& \left.+\left(\sum_{i=1}^{p}\left(\gamma M_{3}\left(\Sigma_{T}-\overline{\Sigma_{T}}\right)\right)_{i}^{2}\right)^{\frac{1}{2}}\right)+C \gamma_{0}\left\|\mathbf{u}_{h}-\overline{\mathbf{u}_{h}}\right\|_{1, \Omega}^{2}-\int_{\Gamma_{C}} \gamma\left(\overline{\lambda_{H t}}-\lambda_{H t}\right)^{2} d \Gamma \\
\lesssim & \mu\left\|G_{N}-\overline{G_{N}}\right\|_{\mathbb{R}^{p}}\left(\left\|U_{T}-\overline{U_{T}}\right\|_{\mathbb{R}^{p}, M_{1}}+\gamma\left\|\Lambda_{T}-\overline{\Lambda_{T}}\right\|_{\mathbb{R}^{p}, M_{2}}+\gamma\left\|\Sigma_{T}-\overline{\Sigma_{T}}\right\|_{\mathbb{R}^{p}, M_{3}}\right) \\
& +C \gamma_{0}\left\|\mathbf{u}_{h}-\overline{\mathbf{u}_{h}}\right\|_{1, \Omega}^{2}-\int_{\Gamma_{C}} \gamma\left(\overline{\lambda_{H t}}-\lambda_{H t}\right)^{2} d \Gamma .
\end{aligned}
$$

The notation $\|\cdot\|_{\mathbb{R}^{p}}$ and $\|\cdot\|_{\mathbb{R}^{p}, M_{k}}$ represent norms on $\mathbb{R}^{p}$. As a consequence, there exist constants depending on $\mathrm{h}$ such that:

$$
\begin{aligned}
& \int_{\Gamma_{C}}\left(\overline{\lambda_{H t}}-\lambda_{H t}\right)\left(u_{h t}-\overline{u_{h t}}\right) d \Gamma+\int_{\Gamma_{C}} \gamma\left(\overline{\lambda_{H t}}-\lambda_{H t}-\sigma_{t}\left(\mathbf{u}_{h}\right)+\sigma_{t}\left(\overline{\mathbf{u}_{h}}\right)\right) \sigma_{t}\left(\overline{\mathbf{u}_{h}}-\mathbf{u}_{h}\right) d \Gamma \\
\lesssim & C \gamma_{0}\left\|\mathbf{u}_{h}-\overline{\mathbf{u}_{h}}\right\|_{1, \Omega}^{2}-\int_{\Gamma_{C}} \gamma\left(\overline{\lambda_{H t}}-\lambda_{H t}\right)^{2} d \Gamma \\
& +\mu C_{1}(h)\left\|\gamma^{1 / 2}\left(g_{H n}-\overline{g_{H n}}\right)\right\|_{0, \Gamma_{C}}\left\|u_{h t}-\overline{u_{h t}}\right\|_{0, \Gamma_{C}} \\
& +\mu C_{2}(h) \gamma_{0}\left\|\gamma^{1 / 2}\left(g_{H n}-\overline{g_{H n}}\right)\right\|_{0, \Gamma_{C}}\left\|\gamma^{1 / 2}\left(\lambda_{H t}-\overline{\lambda_{H t}}\right)\right\|_{0, \Gamma_{C}} \\
& +\mu C_{3}(h) \gamma_{0}\left\|\gamma^{1 / 2}\left(g_{H n}-\overline{g_{H n}}\right)\right\|_{0, \Gamma_{C}}\left\|h_{\Gamma_{C}}^{1 / 2}\left(\sigma_{t}\left(\mathbf{u}_{h}\right)-\sigma_{t}\left(\overline{\mathbf{u}_{h}}\right)\right)\right\|_{0, \Gamma_{C}} \\
\lesssim & C \gamma_{0}\left\|\mathbf{u}_{h}-\overline{\mathbf{u}_{h}}\right\|_{1, \Omega}^{2}-\left\|\gamma^{1 / 2}\left(\lambda_{H t}-\overline{\lambda_{H t}}\right)\right\|_{0, \Gamma_{C}}^{2}+\mu C_{4}(h)\left(1+\gamma_{0}\right)\left\|\gamma^{1 / 2}\left(g_{H n}-\overline{g_{H n}}\right)\right\|_{0, \Gamma_{C}}\left\|\mathbf{u}_{h}-\overline{\mathbf{u}_{h}}\right\|_{1, \Omega} \\
& +\mu C_{2}(h) \gamma_{0}\left\|\gamma^{1 / 2}\left(g_{H n}-\overline{g_{H n}}\right)\right\|_{0, \Gamma_{C}}\left\|\gamma^{1 / 2}\left(\lambda_{H t}-\overline{\lambda_{H t}}\right)\right\|_{0, \Gamma_{C}}
\end{aligned}
$$

where the trace theorem and (3.3) have been used. 
Furthermore, by using Young's inequality we have for any $\beta>0$ :

$$
\begin{aligned}
& \int_{\Gamma_{C}}\left(\overline{\lambda_{H t}}-\lambda_{H t}\right)\left(u_{h t}-\overline{u_{h t}}\right) d \Gamma+\int_{\Gamma_{C}} \gamma\left(\overline{\lambda_{H t}}-\lambda_{H t}-\sigma_{t}\left(\mathbf{u}_{h}\right)+\sigma_{t}\left(\overline{\mathbf{u}_{h}}\right)\right) \sigma_{t}\left(\overline{\mathbf{u}_{h}}-\mathbf{u}_{h}\right) d \Gamma \\
\lesssim & C \gamma_{0}\left\|\mathbf{u}_{h}-\overline{\mathbf{u}_{h}}\right\|_{1, \Omega}^{2}-\left\|\gamma^{1 / 2}\left(\lambda_{H t}-\overline{\lambda_{H t}}\right)\right\|_{0, \Gamma_{C}}^{2}+\beta \mu^{2} \gamma_{0}^{2} C_{5}(h)\left\|\gamma^{1 / 2}\left(g_{H n}-\overline{g_{H n}}\right)\right\|_{0, \Gamma_{C}}^{2} \\
& +\frac{1}{4 \beta}\left\|\gamma^{1 / 2}\left(\lambda_{H t}-\overline{\lambda_{H t}}\right)\right\|_{0, \Gamma_{C}}^{2}+\beta \mu^{2} C_{6}(h)\left(1+\gamma_{0}\right)^{2}\left\|\gamma^{1 / 2}\left(g_{H n}-\overline{g_{H n}}\right)\right\|_{0, \Gamma_{C}}^{2}+\frac{1}{4 \beta}\left\|\mathbf{u}_{h}-\overline{\mathbf{u}_{h}}\right\|_{1, \Omega}^{2} \\
\lesssim & C \gamma_{0}\left\|\mathbf{u}_{h}-\overline{\mathbf{u}_{h}}\right\|_{1, \Omega}^{2}+\beta \mu^{2} \gamma_{0}^{2} C_{5}(h)\left\|\gamma^{1 / 2}\left(g_{H n}-\overline{g_{H n}}\right)\right\|_{0, \Gamma_{C}}^{2} \\
& +\beta \mu^{2} C_{6}(h)\left(1+\gamma_{0}\right)^{2}\left\|\gamma^{1 / 2}\left(g_{H n}-\overline{g_{H n}}\right)\right\|_{0, \Gamma_{C}}^{2}+\frac{1}{4 \beta}\left\|\mathbf{u}_{h}-\overline{\mathbf{u}_{h}}\right\|_{1, \Omega}^{2}
\end{aligned}
$$

From (3.15), (3.13) becomes:

$$
\left\|\mathbf{u}_{h}-\overline{\mathbf{u}_{h}}\right\|_{1, \Omega} \lesssim \mu \frac{\sqrt{\left(1+\gamma_{0}\right)^{2} C_{6}(h)+\gamma_{0}^{2} C_{5}(h)}}{\sqrt{1-2 \gamma_{0}}}\left\|\gamma^{1 / 2}\left(g_{H n}-\overline{g_{H n}}\right)\right\|_{0, \Gamma_{C}}
$$

Combining (3.16) and (3.7) implies that

$\left\|\gamma^{1 / 2}\left(\lambda_{H n}-\overline{\lambda_{H n}}\right)\right\|_{0, \Gamma_{C}} \lesssim \mu\left(\gamma_{0}^{1 / 2}+\gamma_{0}^{-1 / 2}\right) \frac{\sqrt{\left(1+\gamma_{0}\right)^{2} C_{6}(h)+\gamma_{0}^{2} C_{5}(h)}}{\sqrt{1-2 \gamma_{0}}}\left\|\gamma^{1 / 2}\left(g_{H n}-\overline{g_{H n}}\right)\right\|_{0, \Gamma \delta}$

Hence $\Phi_{H}$ is continuous.

Step 2. Let $\left(\mathbf{u}_{h}, \lambda_{H n}, \lambda_{H t}\right)$ be the solution of $\left(P\left(g_{H n}\right)\right)$. Taking $\mathbf{v}_{h}=\mathbf{u}_{h}$ in (3.2) gives

$$
\begin{aligned}
a\left(\mathbf{u}_{h}, \mathbf{u}_{h}\right)+ & \int_{\Gamma_{C}} \lambda_{H n} u_{h n} d \Gamma+\int_{\Gamma_{C}} \gamma\left(-\lambda_{H n}-\sigma_{n}\left(\mathbf{u}_{h}\right)\right) \sigma_{n}\left(\mathbf{u}_{h}\right) d \Gamma \\
& +\int_{\Gamma_{C}} \lambda_{H t} u_{h t} d \Gamma+\int_{\Gamma_{C}} \gamma\left(-\lambda_{H t}-\sigma_{t}\left(\mathbf{u}_{h}\right)\right) \sigma_{t}\left(\mathbf{u}_{h}\right) d \Gamma=L\left(\mathbf{u}_{h}\right) .
\end{aligned}
$$

According to

$$
\int_{\Gamma_{C}} \lambda_{H n} u_{h n} d \Gamma+\int_{\Gamma_{C}} \gamma\left(-\lambda_{H n}-\sigma_{n}\left(\mathbf{u}_{h}\right)\right) \lambda_{H n} d \Gamma=0
$$

and

$$
\int_{\Gamma_{C}} \lambda_{H t} u_{h t} d \Gamma+\int_{\Gamma_{C}} \gamma\left(-\lambda_{H t}-\sigma_{t}\left(\mathbf{u}_{h}\right)\right) \lambda_{H t} d \Gamma \geq 0
$$

we deduce

$$
a\left(\mathbf{u}_{h}, \mathbf{u}_{h}\right)+\int_{\Gamma_{C}} \gamma \lambda_{H n}^{2} d \Gamma-\int_{\Gamma_{C}} \gamma \sigma_{n}\left(\mathbf{u}_{h}\right)^{2} d \Gamma+\int_{\Gamma_{C}} \gamma \lambda_{H t}^{2} d \Gamma-\int_{\Gamma_{C}} \gamma \sigma_{t}\left(\mathbf{u}_{h}\right)^{2} d \Gamma \leq L\left(\mathbf{u}_{h}\right)
$$

From (3.4) and the continuity of $L($.$) :$

$$
C\left\|\mathbf{u}_{h}\right\|_{1, \Omega}^{2} \leq a\left(\mathbf{u}_{h}, \mathbf{u}_{h}\right)-\int_{\Gamma_{C}} \gamma \sigma_{n}\left(\mathbf{u}_{h}\right)^{2} d \Gamma-\int_{\Gamma_{C}} \gamma \sigma_{t}\left(\mathbf{u}_{h}\right)^{2} d \Gamma \leq L\left(\mathbf{u}_{h}\right) \leq C^{\prime}\left\|\mathbf{u}_{h}\right\|_{1, \Omega} .
$$


So, we get

$$
\left\|\mathbf{u}_{h}\right\|_{1, \Omega} \leq \frac{C^{\prime}}{C}
$$

In other respects

$$
\begin{aligned}
\left\|\gamma^{1 / 2} \lambda_{H n}\right\|_{0, \Gamma_{C}}^{2} & =\int_{\Gamma_{C}} \gamma \lambda_{H n}^{2} d \Gamma=\int_{\Gamma_{C}} \lambda_{H n} u_{h n} d \Gamma-\int_{\Gamma_{C}} \gamma \lambda_{H n} \sigma_{n}\left(\mathbf{u}_{h}\right) d \Gamma \\
& \lesssim\left\|\gamma^{1 / 2} \lambda_{H n}\right\|_{0, \Gamma_{C}} \gamma_{0}^{-1 / 2}\left\|\mathbf{u}_{h}\right\|_{1, \Omega}+\left\|\gamma^{1 / 2} \lambda_{H n}\right\|_{0, \Gamma_{C}} \gamma_{0}^{1 / 2}\left\|h_{\Gamma_{C}}^{1 / 2} \sigma_{n}\left(\mathbf{u}_{h}\right)\right\|_{0, \Gamma_{C}} \\
& \lesssim\left\|\gamma^{1 / 2} \lambda_{H n}\right\|_{0, \Gamma_{C}} \gamma_{0}^{-1 / 2}\left\|\mathbf{u}_{h}\right\|_{1, \Omega}+\left\|\gamma^{1 / 2} \lambda_{H n}\right\|_{0, \Gamma_{C}} \gamma_{0}^{1 / 2}\left\|\mathbf{u}_{h}\right\|_{1, \Omega}
\end{aligned}
$$

That implies

$$
\left\|\gamma^{1 / 2} \lambda_{H n}\right\|_{0, \Gamma_{C}} \lesssim\left(\gamma_{0}^{-1 / 2}+\gamma_{0}^{1 / 2}\right)\left\|\mathbf{u}_{h}\right\|_{1, \Omega} \lesssim\left(\gamma_{0}^{-1 / 2}+\gamma_{0}^{1 / 2}\right) \frac{C^{\prime}}{C}
$$

Finally

$$
\left\|\gamma^{1 / 2} \Phi_{H}\left(g_{H n}\right)\right\|_{0, \Gamma_{C}} \lesssim 1, \quad \forall g_{H n} \in M_{H n},
$$

This boundedness of $\Phi_{H}$ together with the continuity of $\Phi_{H}$ proves that there exists at least a solution of Coulomb's discrete frictional contact problem according to Brouwer's fixed point theorem.

Proposition 5. When $\mu$ and $\gamma_{0}$ are small enough, problem (3.1) admits a unique solution.

Proof. From (3.17), we obtain a mesh size dependent uniqueness result when

$C \mu\left(\gamma_{0}^{1 / 2}+\gamma_{0}^{-1 / 2}\right) \frac{\sqrt{\left(1+\gamma_{0}\right)^{2} C_{6}(h)+\gamma_{0}^{2} C_{5}(h)}}{\sqrt{1-2 \gamma_{0}}}<1$. This result means that uniqueness holds when $\mu$ and $\gamma_{0}$ are small enough.

Remark 6. When $\mu=0$, there is existence and uniqueness of the solution if $\gamma_{0}$ is small enough.

\section{Conclusion}

We adapt the Barbosa-Hughes stabilization technique to the nonlinear small strain elastostatics problem with frictional contact. We have obtained a result of existence and uniqueness for the frictional contact problem in elasticity. Afterwards, we can search the theoretical error estimates for the Coulomb friction model.

The characteristic of the Barbosa-Hughes stabilization method is to circumvent the BabuškaBrezzi inf-sup condition. The advantages of the stabilization method can be exploited whenever the Babuška-Brezzi inf-sup condition is difficult or impossible to obtain. Therefore we want to adapt this technique to the so-called eXtended Finite Element Method for crack problems (see [42]). In [40], we combine the XFEM approach together with the Barbosa-Hughes stabilized formulation following the ideas of [27]. 


\section{Acknowledgements}

This work is supported by "l'Agence Nationale de la Recherche", project ANR-05-JCJC-0182-01.

\section{References}

[1] R.A. Adams. Sobolev spaces, Academic Press, 1975.

[2] P. Alart, A. Curnier. Generalisation of Newton type methods to contact problems with friction, J. Mecan. Theor. Appl., 7 (1988), 67-82.

[3] I. Babuška. The finite element method with Lagrange multipliers, Numer. Math., 20 (1973), 179-192.

[4] H.J.C. Barbosa, T.J.R. Hughes. The finite element method with Lagrange multipliers on the boundary: circumventing the Babuška-Brezzi condition, Comput. Methods Appl. Mech. Engrg., 85 (1991), 109-128.

[5] H.J.C. Barbosa, T.J.R. Hughes. Boundary Lagrange multipliers in finite element methods: error analysis in natural norms, Numer. Math., 62 (1992), 1-15.

[6] H.J.C. Barbosa, T.J.R. Hughes. Circumventing the Babuška-Brezzi condition in mixed finite element approximations of elliptic variational inequalities, Comput. Methods Appl. Mech. Engrg., 97 (1992), 193-210.

[7] R. Becker, P. Hansbo, R. Stenberg. A finite element method for domain decomposition with non-matching grids, Math. Model. Numer. Anal., 37 (2003), 209-225.

[8] Z. Belhachmi, F. Ben Belgacem. Quadratic finite element approximation of the Signorini problem, Math. Comp., 72 (2003), 83-104.

[9] Z. Belhachmi, J.M. Sac-Epée, J. Sokolowski. Mixed finite element methods for smooth domain formulation of crack problems, SIAM J. Numer. Anal., 43 (2005), 1295-1320.

[10] F. Ben Belgacem, Y. Renard. Hybrid finite element methods for the Signorini problem, Math. Comp., 72 (2003), 1117-1145.

[11] S.C. Brenner, L.R. Scott. The mathematical theory of finite element methods, SpringerVerlag, 2002.

[12] F. Brezzi. On the existence, uniqueness and approximation of saddle-point problems arising from Lagrange multipliers, Rev. Franç. Automatique Inform. Rech. Opér., Sér. Rouge Anal. Numér., 8 (1974), 129-151.

[13] F. Brezzi, M. Fortin. Mixed and hybrid finite element methods, Springer, 1991. 
[14] Z. Chen. On the augmented Lagrangian approach to Signorini elastic contact problem, Numer. Math., 88 (2001), 641-659.

[15] P.G. Ciarlet. The finite element method for elliptic problems, in Handbook of Numerical Analysis, eds. P.G. Ciarlet and J.L. Lions, North Holland, 2 (1991), 17-352.

[16] M. Cocou, R. Roccou. Numerical analysis of quasistatic unilateral contact problems with local friction, SIAM J. Numer. Anal., 39 (2001), 1324-1342.

[17] P. Coorevits, P. Hild, M. Hjiaj. A posteriori error control of finite element approximations for Coulomb's frictional contact, SIAM J. Sci. Comput., 23 (2001), 976-999.

[18] P. Coorevits, P. Hild, K. Lhalouani, T. Sassi. Mixed finite element methods for unilateral problems: convergence analysis and numerical studies, Math. Comp., 71 (2002), 1-25.

[19] G. Duvaut. Problèmes unilatéraux en mécanique des milieux continus, in Actes du congrès international des mathématiciens (Nice 1970), Gauthier-Villars, 3 (1971), 71-77.

[20] G. Duvaut, J.L. Lions. Les inéquations en mécanique et en physique, Dunod, 1972.

[21] C. Eck, J. Jarušek. Existence results for the static contact problem with Coulomb friction, Math. Models Meth. Appl. Sci., 8 (1998), 445-468.

[22] C. Eck, J. Jarušek, M. Krbec. Unilateral contact problems: variational methods and existence theorems, Pure and Applied Mathematics, CRC Press, 270 (2005).

[23] W. Han, M. Sofonea. Quasistatic contact problems in viscoelasticity and viscoplasticity, American Mathematical Society, International Press, 2002.

[24] P. Hansbo, C. Lovadina, I. Perugia, G. Sangalli. A Lagrange multiplier method for the finite element solution of elliptic interface problems using nonmatching meshes, Numer. Math., 100 (2005), 91-115.

[25] J. Haslinger. Approximation of the Signorini problem with friction, obeying the Coulomb law, Math. Methods Appl. Sci., 5 (1983), 422-437.

[26] J. Haslinger, I. Hlaváček, J. Nečas. Numerical methods for unilateral problems in solid mechanics, in Handbook of Numerical Analysis, Eds. P.G. Ciarlet and J.-L. Lions, North Holland, 4 (1996), 313-485.

[27] J. Haslinger, Y. Renard. A new fictitious domain approach inspired by the extended finite element method, submitted.

[28] R. Hassani, P. Hild, I. Ionescu, N.D. Sakki. A mixed finite element method and solution multiplicity for Coulomb frictional contact, Comput. Methods Appl. Mech. Engrg., 192 (2003), 4517-4531. 
[29] P. Heintz, P. Hansbo. Stabilized Lagrange multiplier methods for bilateral elastic contact with friction, Comput. Methods Appl. Mech. Engrg., 195 (2006), 4323-4333.

[30] P. Hild. Non-unique slipping in the Coulomb friction model in two-dimensional linear elasticity, Q. Jl. Mech. Appl. Math., 57 (2004), 225-235.

[31] P. Hild. Multiple solutions of stick and separation type in the Signorini model with Coulomb friction, Z. Angew. Math. Mech., 85 (2005), 673-680.

[32] P. Hild, P. Laborde. Quadratic finite element methods for unilateral contact problems, Appl. Numer. Math., 41 (2002), 401-421.

[33] P. Hild, Y. Renard. An error estimate for the Signorini problem with Coulomb friction approximated by the finite elements, SIAM J. Numer. Anal., 45 (2007), 2012-2031.

[34] P. Hild, Y. Renard. A stabilized Lagrange multiplier method for the finite element approximation of contact problems in elastostatics, submitted.

[35] J. Jarušek. Contact problems with bounded friction. Coercive case, Czechoslovak. Math. J., 33 (1983), 237-261.

[36] N. Kikuchi, J.T. Oden. Contact problems in elasticity : a study of variational inequalities and finite element methods, SIAM, 1988.

[37] D. Kinderlehrer, G. Stampacchia. An introduction to variational inequalities and their applications, Pure and Applied mathematics, Academic Press, New York-London, 1980.

[38] T. Laursen. Computational contact and impact mechanics, Springer, 2002.

[39] J.-L. Lions, E. Magenes. Problèmes aux limites non homogènes, Dunod, 1968.

[40] V. Lleras. Thesis, in preparation.

[41] V.G. Maz'ya, T.O. Shaposhnikova. Theory of multipliers in spaces of differentiable functions, Pitman, 1985.

[42] N. Moës, J. Dolbow, T. Belytschko. A finite element method for cracked growth without remeshing, Int. J. Numer. Meth. Engng., 46 (1999), 131-150.

[43] J. Nečas, J. Haslinger, J. Jarušek. On the solution of the variational inequality to the Signorini problem with small friction, Bolletino U. M. I., 17 (1980), 796-811.

[44] J. Nitsche. Über ein Variationsprinzip zur Lösung von Dirichlet-Problemen bei Verwendung von Teilräumen, die keinen Randbedingungen unterworfen sind, Abh. Math. Univ. Hamburg, 36 (1971), 9-15.

[45] D.R.J. Owen, D. Peric. Computational model for 3D contact problems with friction based on the penalty method, Int. J. Num. Meth. Eng., 35 (1992), 1289-1309. 
[46] Y. Renard. A uniqueness criterion for the Signorini problem with Coulomb friction, SIAM J. Math. Anal., 38 (2006), 452-467.

[47] M. Shillor, M. Sofonea, J.J. Telega. Models and analysis of quasistatic contact. Variational methods, Springer, 2004.

[48] R. Stenberg. On some techniques for approximating boundary conditions in the finite element method, J. Comput. Appl. Math., 63 (1995), 139-148.

[49] P. Wriggers. Computational Contact Mechanics, Wiley, 2002. 